\title{
A yeast's eye view of mammalian reproduction: cross-species gene co-expression in meiotic prophase
}

Yunfei Li ${ }^{1,3}$, Ka-sum Lam ${ }^{1}$, Nairanjana Dasgupta ${ }^{3}$, Ping Y $\mathrm{e}^{1,2^{*}}$

\begin{abstract}
Background: Meiotic prophase is a critical stage in sexual reproduction. Aberrant chromosome recombination during this stage is a leading cause of human miscarriages and birth defects. However, due to the experimental intractability of mammalian gonads, only a very limited number of meiotic genes have been characterized. Here we aim to identify novel meiotic genes important in human reproduction through computational mining of crossspecies and cross-sex time-series expression data from budding yeast, mouse postnatal testis, mouse embryonic ovary, and human fetal ovary.

Results: Orthologous gene pairs were ranked by order statistics according to their co-expression profiles across species, allowing us to infer conserved meiotic genes despite obvious differences in cellular synchronicity and composition in organisms. We demonstrated that conserved co-expression networks could successfully recover known meiotic genes, including homologous recombination genes, chromatin cohesion genes, and genes regulating meiotic entry. We also showed that conserved co-expression pairs exhibit functional connections, as evidenced by the annotation similarity in Gene Ontology and overlap with physical interactions. More importantly, we predicted six new meiotic genes through their co-expression linkages with known meiotic genes, and subsequently used the genetically more amenable yeast system for experimental validation. The deletion mutants of all six genes showed sporulation defects, equivalent to a $100 \%$ validation rate.
\end{abstract}

Conclusions: We identified evolutionarily conserved gene modules in meiotic prophase by integrating crossspecies and cross-sex expression profiles from budding yeast, mouse, and human. Our co-expression linkage analyses confirmed known meiotic genes and identified several novel genes that might be critical players in meiosis in multiple species. These results demonstrate that our approach is highly efficient to discover evolutionarily conserved novel meiotic genes, and yeast can serve as a valuable model system for investigating mammalian meiotic prophase.

\section{Background}

Meiosis is essential for sexual reproduction in eukaryotes. It is a conserved process in which diploid cells undergo one round of DNA replication followed by two rounds of chromosome segregation to produce haploid cells. Meiosis I separates homologous chromosomes, while meiosis II is similar to mitosis, involving separation of sister chromatids to form haploid cells. Meiosis I

\footnotetext{
* Correspondence: pye@wsu.edu

'School of Molecular Biosciences, Washington State University, PO Box 647520, Pullman, WA 99164, USA

Full list of author information is available at the end of the article
}

and II are, in turn, each divided into four stages: prophase, metaphase, anaphase, and telophase [1].

General chromosome behavior during meiosis is conserved in a range of organisms from unicellular budding yeast to multi-cellular mammals $[1,2]$. However, the time frame required for each meiotic stage varies greatly by sex and species (Figure 1). Budding yeast with heterozygosity at the mating-type locus can finish meiosis in hours under a nutrient-depleted environment [3-5]. Whereas in mammals, germ cells in gonads may take from days to decades to accomplish meiosis with the support of neighboring somatic cells through hormonal cues [6-9]. Male meiosis occurs continuously and

\section{Biomed Central}




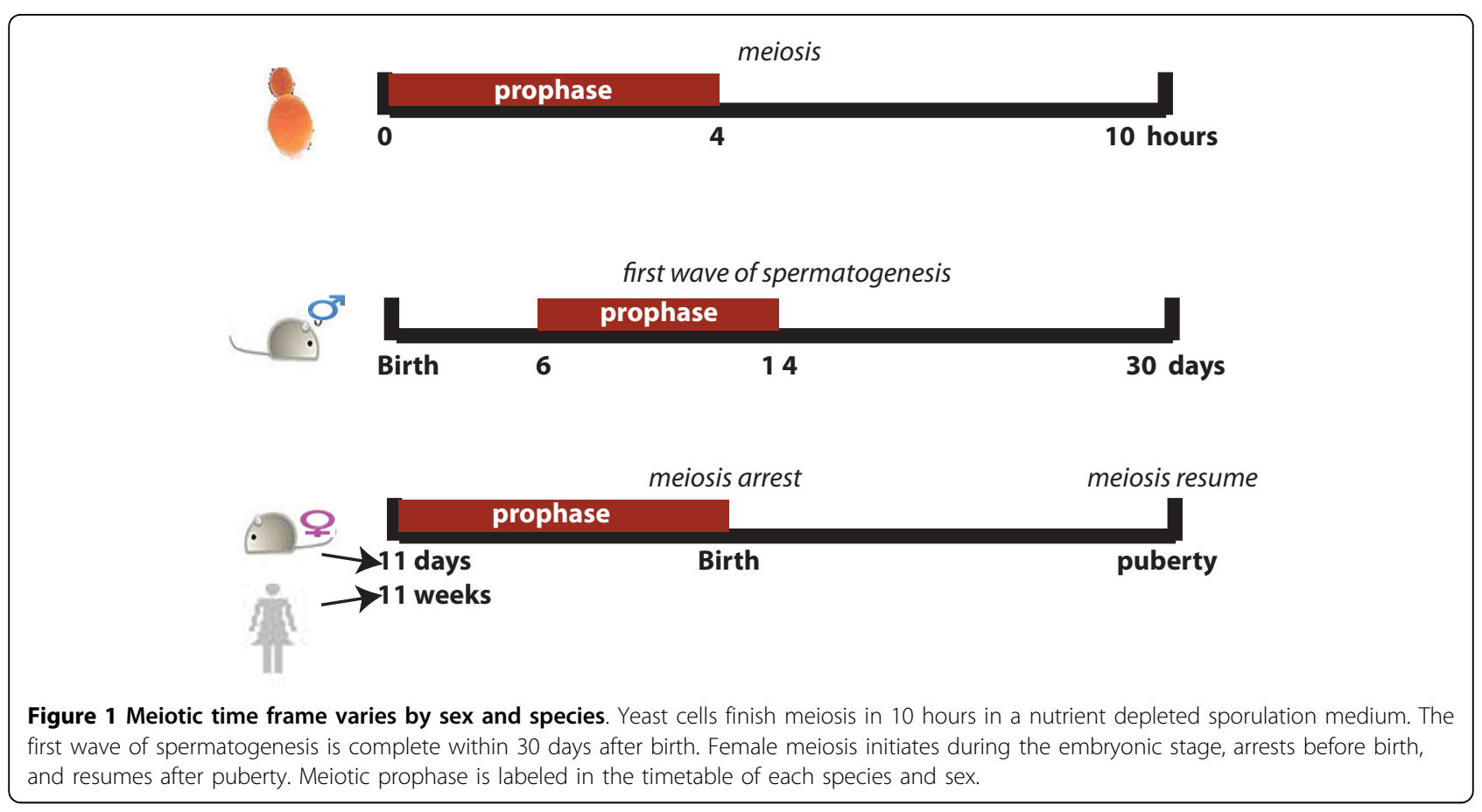

asynchronously from puberty. However, the first wave of spermatogenesis proceeds in a relatively synchronous fashion, which provides the perfect time to investigate genetic control in male meiosis $[7,8]$. In females, the entire oogonial population initiates meiosis synchronously in fetal ovaries and becomes arrested near the end of prophase I before birth. A small cohort of arrested oocytes then resumes meiosis during each ovulation after puberty [6,9-11]. Therefore, prophase I is the most synchronized stage of female meiosis.

The important function of meiosis is to employ recombination to generate genetic diversity in offspring. This process happens during meiotic prophase I (abbreviated as meiotic prophase in the rest of the paper). Each pair of homologous chromosomes aligns together, allowing genetic recombination to occur between nonsister chromatids. The exchanged DNA fragments result in new genetic combinations within chromosomes that will be passed to haploid cells [2]. Recombination errors cause mis-segregation of chromosomes and the production of aneuploid gametes, which are associated with human birth defects and miscarriages. Indeed, alterations in maternal meiotic recombination are an important contributor to both autosomal and sex chromosome trisomies in humans [12]. Many genes involved in meiotic prophase have been characterized in yeast [1]. But comparatively few mammalian genes are known to be involved in this process, and are mainly identified through gene targeting in mice and chromosomal analysis in patients with fertility disorders.
Preliminary studies suggest that humans follow the yeast paradigm, with the early appearance of recombination and cohesion proteins during prophase [13,14].

The recent availability of reproductive tissue-specific expression profiles for humans and mice allows us to monitor gene co-expression and predict plausible new meiotic genes that are important in human reproduction $[6-9,15,16]$. However, the analyses have been mainly limited to simple clustering of expression profiles, which could pinpoint many meiotic gene candidates. Moreover, in vivo mammalian genetics is time-consuming to validate candidate meiotic genes. Here we propose to use budding yeast as a model system with which to identify conserved meiotic genes by applying an order statistics ranking method. Previous computational efforts have demonstrated the feasibility of identifying evolutionarily conserved functional modules through the mining of gene co-expressions, protein interactions, or phenotypes across species [17-19]. Stuart et al described the use of gene co-expression and metagenes to identify conserved genetic modules in humans, flies, worms, and yeast [19]. In this study, we conducted cross-species and cross-sex inferences to identify conserved co-expressed genes in meiotic prophase from time-series expression profiles in yeast, mice, and humans. We identified known meiotic genes from co-expression networks and predicted candidate meiotic genes from co-expression linkages with known meiotic genes. Several novel meiotic genes were subsequently validated in the tractable yeast system. We also examined conserved co-expression pairs using 
enriched genomic information in yeast. Our approach yielded novel genes during the critical meiotic prophase of sexual reproduction and provided insights into the molecular events leading to human reproductive defects.

\section{Results}

Construction of conserved gene co-expression networks for meiotic prophase

There are at least two major challenges to studying meiotic prophase in mammals: the limited amount of available neonatal testis and fetal ovarian tissue and the limited number of techniques for manipulating germ cells in vitro. Microarray is one of the few high-throughput approaches that can provide valuable resources for probing meiotic pathways and networks. The critical issue is germ cell synchrony. Here, we focus on meiotic prophase in the first wave of spermatogenesis and in embryonic oogenesis, which is the most synchronized meiotic process. To construct a conserved co-expression network for meiotic prophase, we analyzed four timeseries microarray studies in yeast $(\mathrm{Y})$, mouse postnatal testis $\left(\mathrm{M}_{\mathrm{m}}\right)$, mouse embryonic ovary $\left(\mathrm{M}_{\mathrm{f}}\right)$, and human fetal ovary $\left(\mathrm{H}_{\mathrm{f}}\right)$ (Figure 2) $[5,6,8,9]$.

We define metagenes as genes conserved across multiple species [19]. We derived three types of metagenes: metagenes conserved across yeast, mice, and humans (YMH), and metagenes conserved only between two species (YM and $\mathrm{YH}$ ) (Table 1, Figure 2). Every metagene type contains yeast genes. Thus, we can examine conserved co-expression genes using the enormous amount of genomic data available on yeast and validate predicted genes using yeast as a tractable experimental system.

The use of metagenes allows us to connect gene expression profiles across species. To identify conserved co-expression genes, we first computed the Pearson correlation of gene expression across the prophase time points for metagene pairs in each species. Metagene pairs were subsequently ranked according to Pearson correlation coefficients. A rank ratio was obtained for each metagene pair by dividing its rank by the total number of metagene pairs in the species. Next, we used the joint cumulative distribution of order statistics to evaluate the probability of observing a particular configuration of ranks across different organisms by chance [19]. This P-value quantifies the significance of observing the co-expression of metagene pairs across species. Metagene pairs with P-values greater than a threshold can be connected to form networks. In this way, we constructed four networks that capture gene co-expression during meiotic prophase conserved in different organisms and different sexes: $Y-M_{m}, Y-M_{f}, Y-H_{f}$ and $Y$ $M_{m}-M_{f^{-}} H_{f}$ (Figure 2). A toy example for constructing

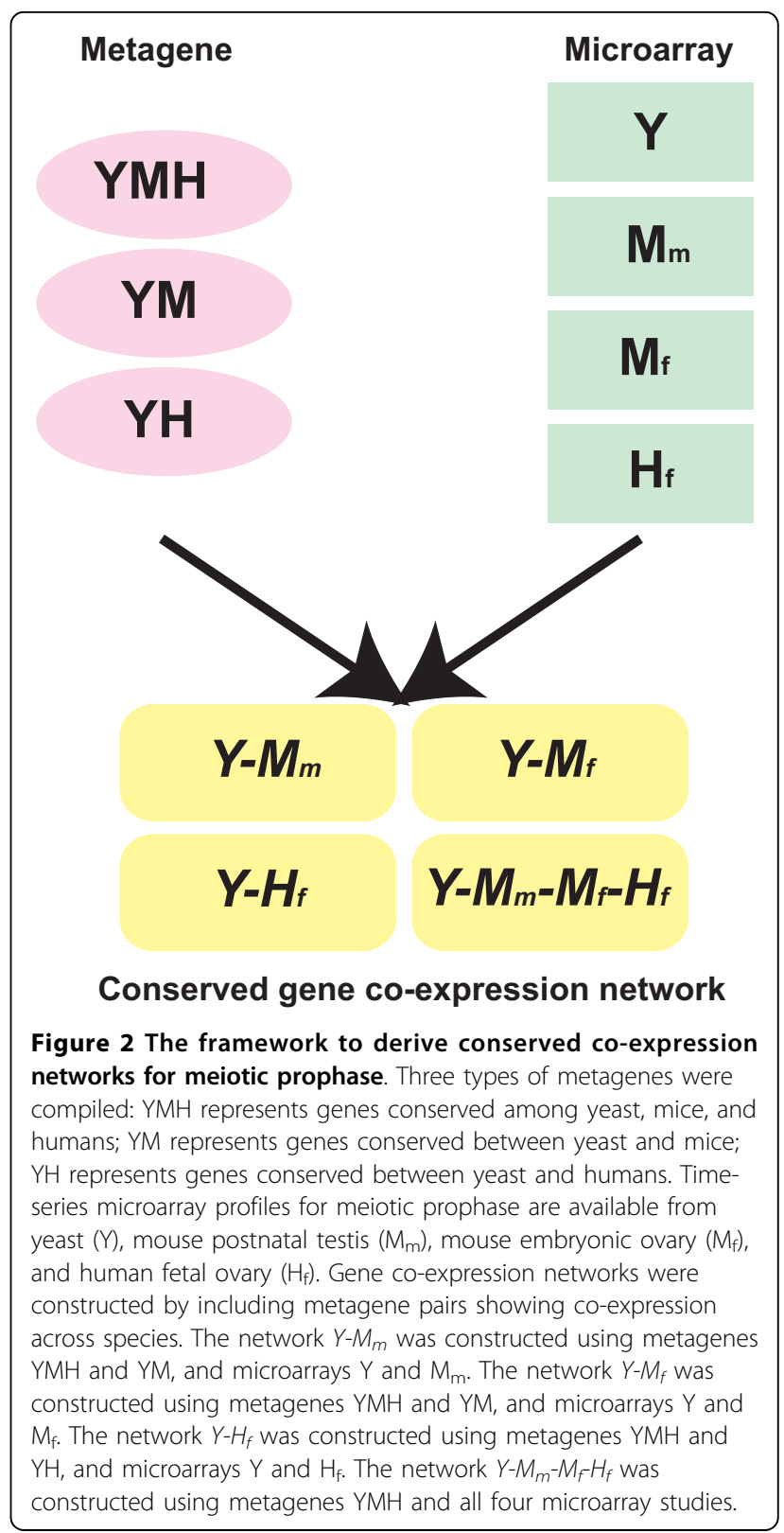

Table 1 The number of conserved genes in yeast, mouse, and human

\begin{tabular}{cccccccc}
\hline \multicolumn{4}{c}{$\begin{array}{c}\text { Metagene } \\
\text { type* }\end{array}$} & & & & \\
\cline { 2 - 4 } & YMH & YM & YH & Sum & $\begin{array}{c}\text { Genome } \\
\text { coverage }\end{array}$ & $\begin{array}{c}\text { Genes per } \\
\text { metagene }\end{array}$ \\
\hline Metagene & 2,036 & 146 & 129 & 2,311 & - & - \\
Yeast & 2,124 & 151 & 131 & 2,406 & $42 \%$ & 1.04 \\
Mouse & 2,121 & 151 & - & 2,272 & $10 \%$ & 1.04 \\
Human & 2,165 & - & 133 & 2,298 & $10 \%$ & 1.06 \\
\hline
\end{tabular}

* YMH, YM, and $\mathrm{YH}$ are mutually exclusive metagene types.

\# The genome coverage was calculated based on total protein numbers in yeast $(5,792)$, mouse $(23,132)$, and human $(22,983)$ [52]. 
gene co-expression networks across species is described in Additional file 1, Figure S1.

\section{Conserved gene co-expression recovers known meiotic genes}

We evaluated conserved co-expression networks using known meiotic genes annotated by gene ontology (GO:0007126) [20]. There are 72 yeast meiotic genes, 19 mouse meiotic genes, and 13 human meiotic genes among all metagenes, reflecting our limited knowledge of conserved meiotic processes in these three species. We plotted precision-coverage curves, which are the standard for determining method performance (Figure 3 ). Precision is defined as the ratio of known meiotic genes to all metagenes. Coverage is the number of known meiotic genes. All metagene pairs in each coexpression network were sorted by the significance of their P-values. We then calculated precision and coverage values in 100-pair increments to plot the curve. Yeast results show that a higher percentage of metagenes are meiotic in the top metagene pairs (Figure 3A). As we go down the list of metagene pairs by P-values, the precision of meiotic genes decreases; this was true for all four networks containing yeast genes. Interestingly, the top-100 metagene pairs in the network $Y-M_{m}$ contain a higher fraction of meiotic genes than the other three networks $Y-M_{f}, Y-H_{f}$, and $Y-M_{m}-M_{f}-H_{f}$. This suggests that yeast meiosis might be more similar to the male mouse process than to the female process. Note that the low precision rate (y-axis) is not due to the computational method, but results from the very small number of known meiotic genes ( $x$-axis) among all the genes in the networks.

When we used mouse and human meiotic genes to evaluate co-expression networks, we saw results similar to those in yeast (Figure 3). Top metagene pairs contain a higher fraction of meiotic genes. Meiotic gene enrichment declines when metagene pairs are ranked down the list. The network $Y-M_{m}$ is more enriched for mouse meiotic genes than $Y-M_{f}$ and $Y-M_{m}-M_{f}-H_{f}$ (Figure 3B), again suggesting the similarity between yeast meiosis and male meiosis.

We further evaluated enriched GO terms among the top-100 metagene pairs from four co-expression networks. We chose top-100 metagene pairs because they are highly enriched for meiotic genes (Figure 3). When using yeast genes to search for GO terms, we found that the meiosis term was significantly enriched in all four networks (P-values $=0.001$ for $Y-M_{m}, 0.019$ for $Y-M_{f}$, 0.009 for $Y-H_{f}, 0.033$ for $Y-M_{m}-M_{f} H_{f}$ ) (Table 2). Consistent with the precision-coverage results, this again indicates that our co-expression networks can efficiently identify known meiotic genes. Yeast genes participating in the cell cycle, in DNA metabolism, and in protein folding are also present in the co-expression networks. When using mouse genes to identify enriched GO terms among the top-100 metagene pairs, we identified many significant terms describing meiotic processes (Additional file 1, Table S1). Synapsis is the pairing of two homologous chromosomes that enables crossover during prophase. This term is enriched in both networks $Y-M_{m}$ and $Y-M_{f}\left(\mathrm{P}\right.$-values $=0.022$ for $Y-M_{m}, 0.001$ for $\left.Y-M_{f}\right)$. Pachytene is the most significant GO term in the

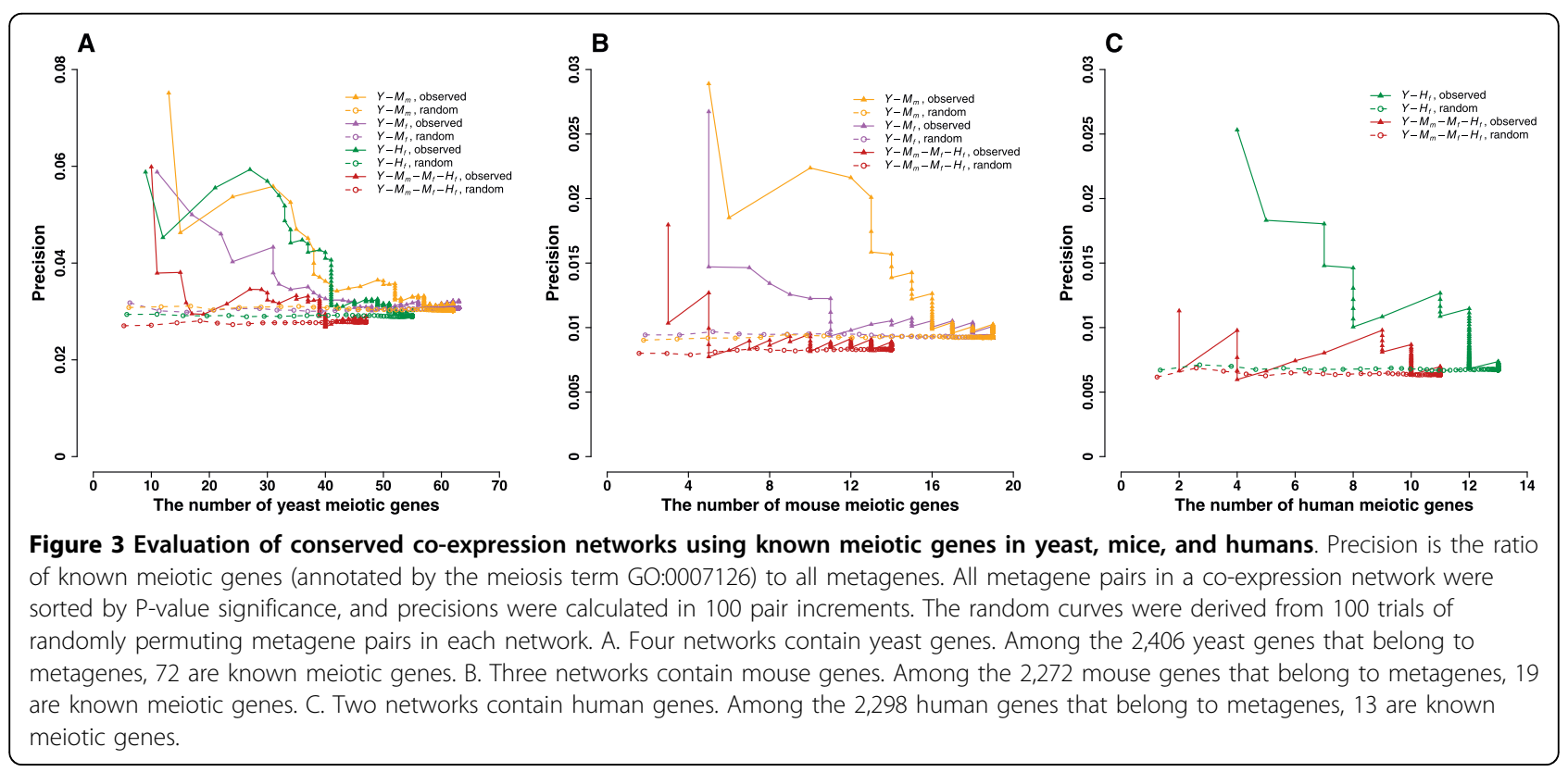




\begin{tabular}{|c|c|c|}
\hline GO Term & GO Name & Hypergeometric P-value \\
\hline \multicolumn{3}{|l|}{$\overline{Y-M_{m}}$} \\
\hline GO:0007126 & meiosis & 0.001 \\
\hline GO:0007049 & cell cycle & 0.006 \\
\hline GO:0006950 & response to stress & 0.025 \\
\hline GO:0006997 & nucleus organization & 0.027 \\
\hline \multicolumn{3}{|l|}{$\overline{Y-M_{f}}$} \\
\hline GO:0007126 & meiosis & 0.019 \\
\hline GO:0007165 & signal transduction & 0.023 \\
\hline GO:0000746 & conjugation & 0.024 \\
\hline GO:0016044 & membrane organization & 0.030 \\
\hline GO:0007049 & cell cycle & 0.037 \\
\hline GO:0007114 & cell budding & 0.044 \\
\hline \multicolumn{3}{|l|}{$\overline{Y-H_{f}}$} \\
\hline GO:0006259 & DNA metabolic process & 0.001 \\
\hline GO:0006996 & organelle organization & 0.004 \\
\hline GO:0007126 & meiosis & 0.009 \\
\hline GO:0007010 & cytoskeleton organization & 0.010 \\
\hline GO:0006350 & transcription & 0.025 \\
\hline GO:0007049 & cell cycle & 0.035 \\
\hline \multicolumn{3}{|l|}{$\overline{Y-M_{m}-M_{f}-H_{f}}$} \\
\hline GO:0006457 & protein folding & 0.031 \\
\hline GO:0007126 & meiosis & 0.033 \\
\hline
\end{tabular}

* Top metagene pairs are the pairs with the most significant P-values for coexpression across species.

\# Significant GO terms are defined by hypergeometric P-value $<0.05$.

network $Y-M_{m}-M_{f}-H_{f}(\mathrm{P}$-value $=0.010)$. It is a stage of meiotic prophase characterized by synapsis and by the occurrence of crossover. Other enriched GO terms related to reproduction include female gamete generation, reciprocal meiotic recombination, DNA recombination, mismatch repair, microtubule-based movement, and so on. When using human genes to identify enriched GO terms among the top-100 metagene pairs (Additional file 1, Table S2), we again identified pachytene as a significant GO term in the network $Y-M_{m}-M_{f}$ $H_{f}(\mathrm{P}$-value $=0.010)$. We also found other enriched GO terms directly associated with reproduction, such as cell cycle, reciprocal meiotic recombination, DNA replication, meiosis, double-strand break repair, female gamete generation, and spermatogenesis.

Next, we determined whether using conservation of co-expression between different species improved the identification of meiotic genes as compared to an alternative approach by using co-expression in a single species. To this end, we sorted metagene pairs by Pearson correlation coefficient in each microarray and plotted precision-coverage curves to evaluate co-expression in individual microarrays using known meiotic genes
(Additional file 1, Figure S2). Interestingly, the conserved co-expression approach showed no improvement in predicting known yeast meiotic genes from top metagene pairs as compared to the co-expression approach in a single species (compare Figure 3A with Additional file 1, Figure S2A). However, the conserved co-expression approach displayed a higher enrichment of known mouse and human meiotic genes among top metagene pairs than the co-expression approach in individual species (compare Figure 3 with Additional file 1, Figure S2B-S2C). For example, only one known human meiotic gene was recovered in the top-100 metagene pairs from female human microarray by using the co-expression approach in individual species (Additional file 1, Figure S2C), while four were recovered from the top-100 metagene pairs by using the conserved co-expression between yeast and human studies (Figure $3 \mathrm{C}$ ). We also identified enriched GO terms among the top-100 metagene pairs from individual microarrays (Additional file 1, Table S3). Similar to the results obtained from precision-coverage curves, the conserved co-expression approach showed no improvement for the yeast study in terms of enrichment of meiosis-related GO terms, but improved the enrichment of mammalian meiosis-related GO terms (compare Additional file 1, Table S3 with Table 2 and Additional file 1, Table S1 and Table S2). Finally, we compared the top-100 metagene pairs from the conserved co-expression approach with the top-100 metagene pairs from the co-expression approach in a single species, and found varied degrees of metagene pair overlap between the two sets, ranging from 0 to 26 . In summary, the conserved co-expression approach can improve the identification of mammalian meiosis genes although the genes identified by this approach do not necessarily overlap with meiotic genes identified by the simple co-expression approach from individual species.

\section{Properties of conserved co-expression genes and gene pairs}

Because all four co-expression networks contain yeast genes, we took advantage of enriched yeast genomic information to investigate the properties of top ranked metagene pairs. We first examined whether significant co-expression links overlapped with protein interactions. Our results show that top co-expression metagene pairs in all four observed networks are more enriched for physical interactions as compared to those in randomized networks. This is evidenced by co-localization in the same MIPS protein complex [21] and by overlap with physical interactions retrieved from the BioGRID database [22] (Figure 4). In particular, top metagene pairs in the most conserved network, $Y-M_{m}-M_{f}-H_{f}$, overlap with a higher fraction of physical interactions than those in the other three co-expression networks. The 

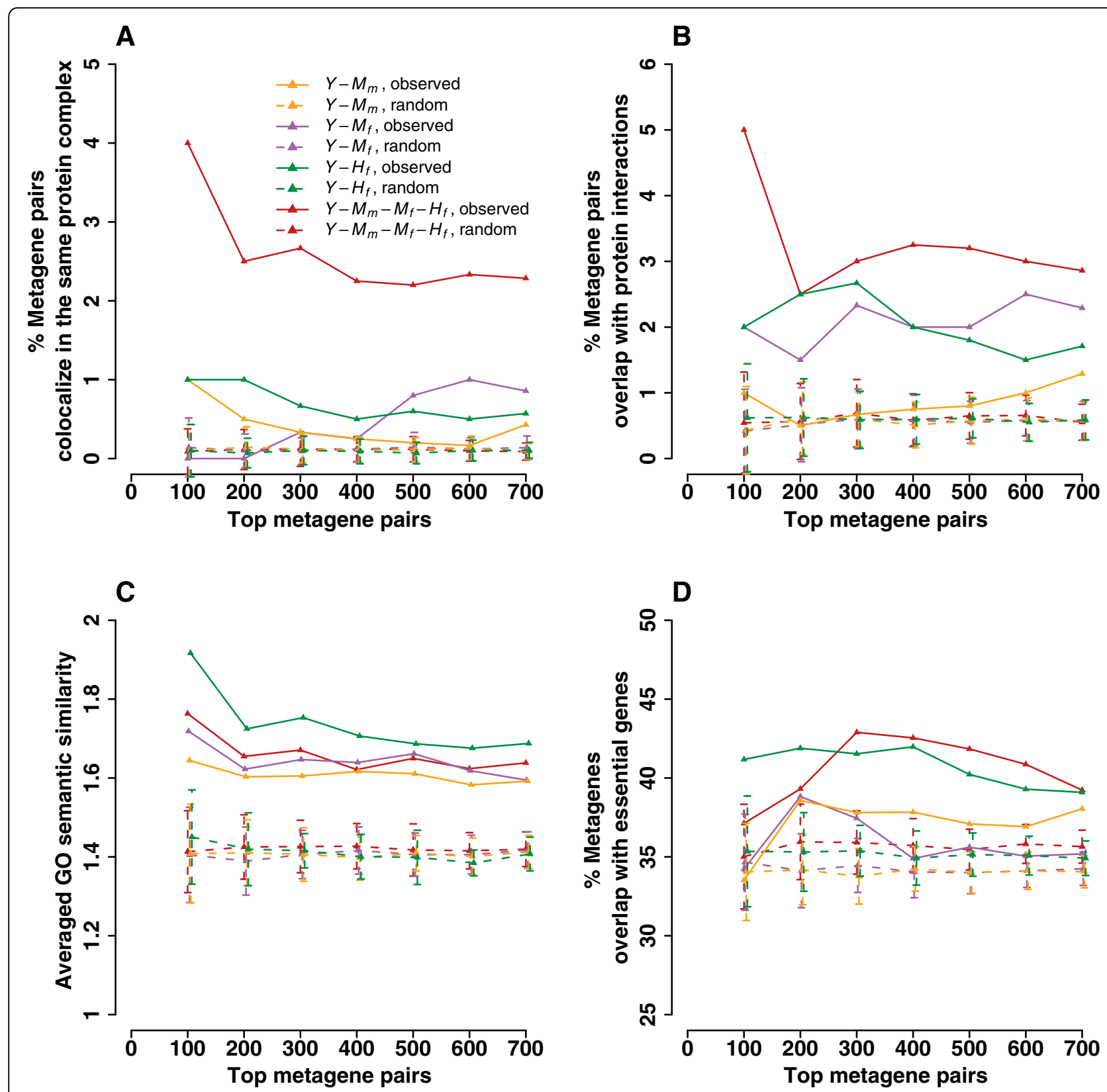

Figure 4 Characterizations of metagene pairs and metagenes using yeast genomic information. Top metagene pairs are the pairs with the most significant P-values for co-expression across species. The random curves with mean and standard deviation labeled were derived from 100 trials of randomly permuting metagene pairs in each network. A. Yeast metagene pairs co-localized in the same protein complex. The observed curve is significantly different from the randomized curve for all networks except $Y$ - $M_{f}$ (Welch Two Sample t-test $P<0.05$ ). B. Yeast metagene pairs overlapping with protein interactions. The observed curve is significantly different from the randomized curve for all four networks (Welch Two Sample t-test $P$ < 0.05). C. Averaged semantic similarity of yeast metagene pairs calculated using GO sub-ontology Biological Process. The observed curve is significantly different from the randomized curve for all four networks (Welch Two Sample t-test $P<0.05)$. D. Yeast metagenes overlapping with essential genes. The observed curve is significantly different from the randomized curve for all four networks (Welch Two Sample t-test $P<0.05$ ).

co-occurrence of protein interaction and expression correlation can suggest high-confidence functional associations of metagene pairs.

Next, we examined the functional associations of yeast metagene pairs using averaged GO semantic similarity in the sub-ontology Biological Process [23]. Biological Process implies a series of molecular events in which a gene participates. We found that the averaged semantic similarity of observed metagene pairs is greater than that of randomized pairs for all four co-expression 
networks (Figure 4C). The top-100 metagene pairs showed the tightest functional connection. This connection declined with increased inclusion of metagene pairs down the significant P-value list.

The yeast genome contains approximately 1,100 essential genes required for cell viability in the rich glucose medium [24]. This is equivalent to $18 \%$ of the total genes. The percentage of metagenes overlapping with essential genes was approximately $35 \%$ for randomized metagene pairs, suggesting evolutionarily conserved metagenes are enriched for essential genes (Figure 4D). Metagenes from the top co-expression pairs contained a slightly higher percentage of essential genes than those from randomized co-expression pairs. This indicates that a significant number of essential proteins may participate in the conserved meiotic process.

We also explored other yeast gene properties of the top metagene pairs in four co-expression networks. In a large-scale parallel phenotypic study, 261 sporulationdeficient genes and 102 sporulation-proficient genes were identified by screening 4,000 yeast deletion strains [25]. However, metagenes from the top co-expression pairs were not enriched for sporulation-proficient or deficient genes in all networks (data not shown). Inconsistent hits from global expression and deletion screens have been reported in many studies [4,24-26], and may be due to gene redundancy or indirect/non transcriptional responses. Synthetic lethality refers to a genetic interaction between two genes that cause cell death when they mutate concurrently, but neither by itself is lethal. The top metagene pairs were not enriched for synthetic lethal interactions (data not shown), although they were enriched for physical interactions. This is consistent with a previous finding that physical interactions imply function in a single pathway and are orthogonal to synthetic lethal interactions $[27,28]$.

To examine mouse and human orthologs in coexpression networks, we compiled a list of mouse protein interactions from five databases (Biomolecular Interaction Network Database, Database of Interacting Proteins, Molecular INTeraction database, IntAct, and BioGrid) [22,29-32], and a list of human protein interactions from five databases (Human Protein Reference Database, Database of Interacting Proteins, Molecular INTeraction database, IntAct, and BioGrid) [22,30-33]. However, very few top-700 metagene pairs from any network overlapped with known human or mouse protein interactions. We believe this is due to the lack of coverage on protein-protein interactions in mammals because we did observe enriched yeast protein interactions among the top metagene pairs (Figure 4). The averaged GO semantic similarity of mouse and human genes showed a trend similar to that of the yeast genes (Figure 4C), with the top-100 pairs exhibiting the tightest functional connections (Additional file 1, Figure S3). Essential genes in humans [34,35] and mice [36] were not over-represented among the top metagene pairs (Additional file 1, Figure S4), which may also be related to mammalian data quality and coverage.

\section{Transcript abundance of conserved co-expression genes}

The gene co-expression was derived from Pearson correlation, which measures similarity of gene expression over time rather than absolute mRNA abundance. Therefore, we were interested in investigating the transcript abundance of metagene pairs in our networks (Figure 5). First, we identified maximal signal intensity for each gene over the course of prophase. Next, we ranked maximal signal intensities genome-wide to identify the median value. Any genes with maximal signal intensity above the median were defined as highly expressed genes, while the rest were defined as lowly expressed genes. Thus, we classified the top-100 metagene pairs in each two-species network as both highly expressed genes, both lowly expressed genes, or one highly expressed and one lowly expressed gene. We chose to analyze top-100 metagene pairs because they are highly enriched for meiotic genes and exhibit close functional connections (Figure 3, Figure 4).

Results show that most mouse and human orthologs of the top metagene pairs were highly expressed (Figure 5). This was demonstrated by the finding that $82 \%$ of the pairs in $Y-M_{m}$ were highly expressed in mouse postnatal testis, $74 \%$ of the pairs in $Y-M_{f}$ were highly expressed in mouse embryonic ovary, and $72 \%$ of the pairs in $Y-H_{f}$ were highly expressed in human fetal ovary. However, the transcript abundance of yeast genes did not follow the same trend as that in mammalian genes. In all three networks, approximately half of the yeast pairs had two highly expressed genes, whereas the other half of the pairs had one highly expressed and one lowly expressed gene. We observed that $Y-M_{m}$ has $46 \%$ metagene pairs that are consistently highly expressed in yeast and male mice (Figure 5A), whereas $Y-M_{f}$ and $Y-H_{f}$ have only $34 \%$ and $36 \%$ pairs consistently highly expressed between yeast and females (Figure 5). This again suggests that yeast meiosis might more closely resemble the male process than the female process.

To differentiate yeast metagene pairs with two highly expressed genes from those with one highly and one lowly expressed gene, we calculated the hypergeometric P-values of enriched GO terms for these two groups of yeast metagene pairs [20]. We found that the meiosis term (GO:0007126) was significantly enriched among metagene pairs with both genes highly expressed (P-values $=0.0003$ for $Y-M_{m}, 0.05$ for $Y-M_{f}, 0.005$ for $\left.Y-H_{f}\right)$. Indeed, meiosis is the most significant GO term for networks $Y-M_{m}$ and $Y-H_{f}$. By contrast, the meiosis 

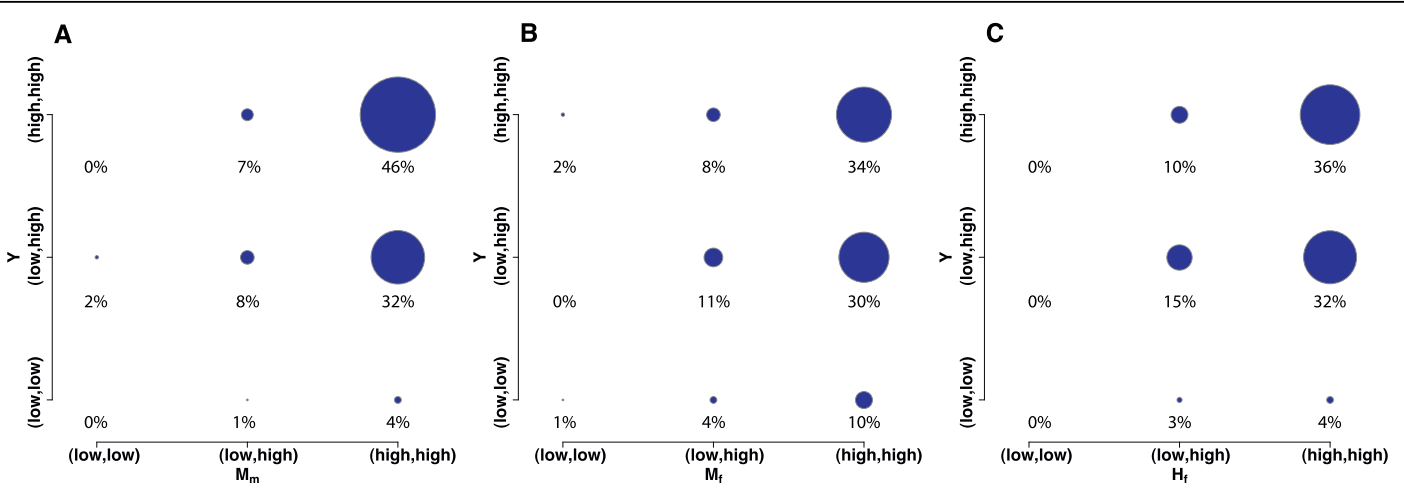

Figure 5 Transcript abundance of genes in three two-species co-expression networks. Top-100 metagene pairs with the most significant co-expression P-values were used for plotting the figure. The transcript abundance of genes from the metagene pairs was identified. The (low, low) means both genes have mRNA abundance below the median of genome-wide signal intensities, the (low, high) means one gene has mRNA abundance below the median of genome-wide signal intensities, with the other above the median, and the (high, high) means both genes have mRNA abundance above the median of genome-wide signal intensities. The size of circles corresponds to the number of metagene pairs. A. $Y-M_{m}$ network. B. $Y-M_{f}$ network. C. $Y-H_{f}$ network.

term was not enriched in the yeast metagene pairs with one highly expressed and one lowly expressed gene in all three networks. This suggests that most yeast meiotic genes are highly expressed during prophase. In fact, these highly expressed yeast meiotic genes in prophase are generally highly expressed throughout meiosis when we define the expression level based on the entire meiosis time course.

\section{Prediction of novel meiotic genes in prophase}

We have demonstrated that conserved co-expression networks can successfully recover known meiotic genes (Figure 3). We have also shown that conserved coexpression pairs exhibit close functional connections, as evidenced by the GO annotation similarity and overlap with physical interactions (Figure 4). Therefore, we can predict novel meiotic genes through their co-expression linkages with known meiotic genes. More accurate predictions can be attained when co-expression linkages overlap with physical interactions or annotation similarity. These candidate meiotic genes can first be experimentally validated using a genetically tractable yeast system before being tested in a mammalian system.

Four co-expression networks, $Y-M_{m}, Y-M_{f}, Y-H_{f}$, and $Y-M_{f^{-}} M_{m}-H_{f}$, were constructed by linking the top-100 metagene pairs. We focus on top-100 metagene pairs because they are most enriched for meiotic genes and exhibit close functional connections (Figure 3, Figure 4). The top-100 metagene pairs in each network are listed in the Additional file 1, Table S4; many of these pairs are common to more than one network (Table 3). To identify meiotic prophase gene modules, we isolated gene clusters from networks containing yeast meiotic genes, sporulation-deficient genes, or sporulation-proficient genes (Figure 6) [20,25]. Because yeast is present in every network, we used yeast gene names to label the metagenes. Human and mouse orthologs of yeast genes can be easily mapped from metagenes (Table 4). Properties of yeast genes and their interactions were labeled onto these modules to facilitate interpretations, including essential genes, protein interactions, synthetic lethal interactions, and protein complexes [21,22,24].

The time frame of microarray data used in our study covers meiotic prophase. Consistently, we observed the appearance of homologous recombination proteins and chromatin cohesion proteins in all four networks (Figure 6). These genes include HOP1, DMC1, SPO11, SPO22, $M S H 4, M S H 5$, and SCC2. The co-expression link between $H O P 1$ and $D M C 1$ was shown in networks $Y-H_{f}$ and $Y-M_{m}-M_{f^{-}} H_{f} . H O P 1$ encodes for a meiosis-specific DNA binding protein and is required for homologous chromosome pairing. DMC1 encodes for a strand invasion protein, an essential component of the meiotic homologous recombination machinery [2]. The connection between HOP1 and DMC1 suggests the coordinated events of chromosome pairing and recombination. In the $Y-H_{f}$ network, $H O P 1$ and $D M C 1$ were further linked to SPO11, SPO22, MSH5, and SCC2. These genes exhibit coherent functions and are located in the same cluster with both direct and indirect links. SPO11 initiates meiotic recombination by catalyzing the formation of double-strand breaks in DNA, while SPO22 is a meiosisspecific gene essential for chromosome pairing [1]. Msh4 and Msh5 are meiotic recombination proteins and form heterodimers [2]. They are connected by both coexpression and protein interaction in networks $Y-M_{m}$ and $Y-M_{f} . M S H 5$ also showed consistent co-expression links with $S C C 2$ in networks $Y-M_{m}, Y-H_{f}$, and $Y-M_{m^{-}}$ $M_{f}-H_{f} . S C C 2$ is a cohesion loading factor involved in establishing sister chromatid cohesion during double 
Table 3 The top-100 metagene pairs* common to at least two conserved co-expression networks

\begin{tabular}{|c|c|c|c|c|}
\hline Top-100 metagene pairs & $Y-M_{m}$ & $Y-M_{f}$ & $Y-H_{f}$ & $Y-M_{m}-M_{f} H_{f}$ \\
\hline DED1-RTS2 & + & + & + & + \\
\hline CDC28-CUP5 & + & + & + & + \\
\hline NIF3-RIM15 & + & + & + & + \\
\hline RRI1-TID3 & + & + & + & \\
\hline PUT1-YOR289W & + & + & + & \\
\hline CDC33-UNG1 & + & + & + & \\
\hline DUS3-MDJ1 & + & + & + & \\
\hline BRF1-LSC2 & + & + & + & \\
\hline BUD32-FET5 & + & & + & + \\
\hline MSH5-SCC2 & + & & + & + \\
\hline GET3-SRP102 & & + & + & + \\
\hline DOC1-MSH4 & + & + & & \\
\hline MSH4-MSH5 & + & + & & \\
\hline PRE1-SMC6 & + & + & & \\
\hline MLP1-YFR018C & + & + & & \\
\hline GOR1-YPL225W & + & + & & \\
\hline AGX1-YMR074C & + & & + & \\
\hline PYC1-SSU72 & + & & + & \\
\hline MCM2-RRN3 & & + & + & \\
\hline CCT5-YKE2 & + & & & + \\
\hline COG3-CYB5 & + & & & + \\
\hline COG6-HOP1 & + & & & + \\
\hline DPP1-KAP114 & + & & & + \\
\hline NAM8-STE24 & + & & & + \\
\hline PTC3-YEF1 & + & & & + \\
\hline RPL32-RPL37A & + & & & + \\
\hline CDC50-RRP1 & + & & & + \\
\hline PUFG-RVB1 & + & & & + \\
\hline PNG1-SPT15 & + & & & + \\
\hline MTC5-TLG1 & + & & & + \\
\hline$A S C 1-R P P 2 B$ & & + & & + \\
\hline APT1-ATP16 & & + & & + \\
\hline DIA4-SRP72 & & + & & + \\
\hline GOS1-RIM11 & & + & & + \\
\hline FOX2-HRD1 & & + & & + \\
\hline HRD1-RPN1 & & + & & + \\
\hline IFM1-MTO1 & & + & & + \\
\hline KAR3-RPN7 & & + & & + \\
\hline FMP32-LSM12 & & + & & + \\
\hline NFU1-RBG1 & & + & & + \\
\hline RPA135-VPS15 & & + & & + \\
\hline BZZ1-PRP16 & & & + & + \\
\hline DMC1-HOP1 & & & + & + \\
\hline IDH2-UTP4 & & & + & + \\
\hline MAK10-MAK5 & & & + & + \\
\hline CGI121-NIF3 & & & + & + \\
\hline CUL3-PCM1 & & & + & + \\
\hline QRI1-RPN2 & & & + & + \\
\hline RAD27-RPT6 & & & + & + \\
\hline RPN7-SDH2 & & & + & + \\
\hline
\end{tabular}

Table 3 The top-100 metagene pairs* common to at least two conserved co-expression networks (Continued)

\begin{tabular}{lcc}
\hline POB3-SCP160 & + & + \\
DDP1-SPB1 & + & + \\
TLG1-TYW3 & + & + \\
YBR241C-YNL155W & + & + \\
\hline
\end{tabular}

* Top metagene pairs are the pairs with the most significant P-values for coexpression across species.

strand break repair [37]. This indicates that regulation of sister chromatid cohesion is synchronized with recombination events during meiotic prophase. Interestingly, we observed that $D O C 1$ is always associated with a cluster of $\mathrm{MSH} 4, \mathrm{MSH} 5$, and $\mathrm{SCC} 2$ in the three, twospecies networks. Yeast Doc1 and its mammalian ortho$\log$ APC10 are a subunit of the anaphase promoting complex, a conserved ubiquitin ligase complex that degrades mitotic cyclins and anaphase inhibitory proteins, thereby triggering sister chromatid separation and exit from mitosis [38,39]. However, the involvement of $D O C 1$ in the meiotic process has never been shown before. The co-expression link with recombination and cohesion proteins indicates that $D O C 1$ might also function during meiotic prophase.

NAM8 is a component of the U1 snRNP protein involved in the formation of double strand breaks [40]. The nam 8 deletion mutant is defective in sporulation [25]. A co-expression link between NAM8 and STE24 was observed in $Y-M_{m}$ and $Y-M_{m}-M_{f}-H_{f}$. STE24 is a highly conserved zinc metalloprotease that functions in two steps of a-factor maturation in yeast [41]. Its human ortholog, $F A C E 1$, is highly expressed in testis and ovary [42]. The linkage between NAM8 and STE24 suggests a possible new role for STE24 in meiotic recombination. Furthermore, an uncharacterized ORF $Y D L 114 \mathrm{~W}$ is also connected to STE24 in $Y-M_{m}$, and to the essential transporter gene SED5 [43] in $Y-M_{f} . Y D L 114 W$ is predicted to have peptide transporter activity, possibly related to the fact that a-factor is a secreted peptide. Another uncharacterized ORF, YDR374C, exhibited a co-expression linkage with PMS1 in the network $Y-M_{f}$. PMS1 encodes for an ATP-binding protein and is required for mismatch repair in meiosis [44]. This linkage predicts a possible role for $Y D R 374 C$ in meiotic recombination.

The general notion about meiosis conservation is that entry signaling has diverged substantially among species but mechanical components and enzymes are conserved [2]. For example, yeast cells initiate meiosis in a nutrient depleted sporulation medium, while mammalian germ cells initiate meiosis in response to extrinsic inducer retinoic acid $[3,10,11]$. However, our findings suggest potential players that might control the conserved meiotic entry process. We identified two genes, RIM11 and 


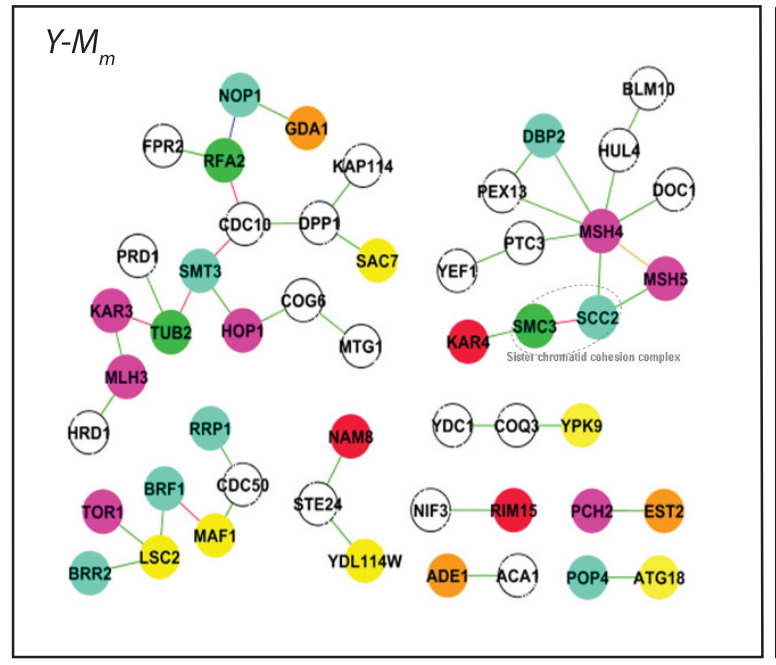

C
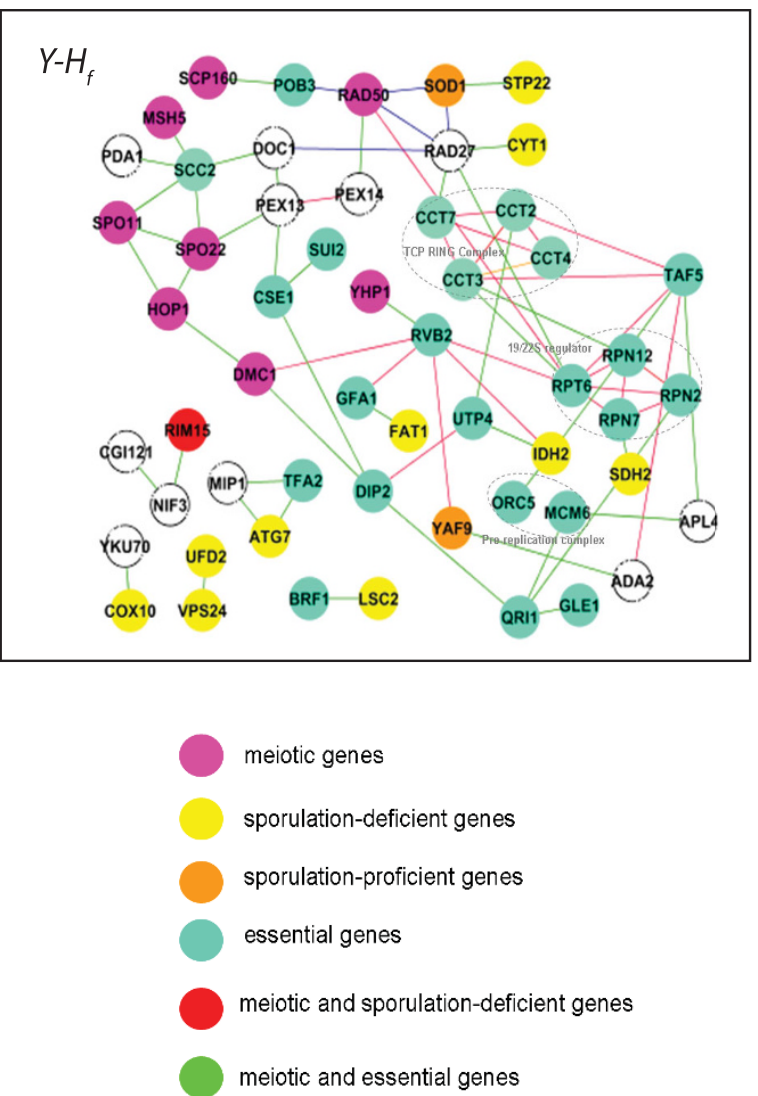

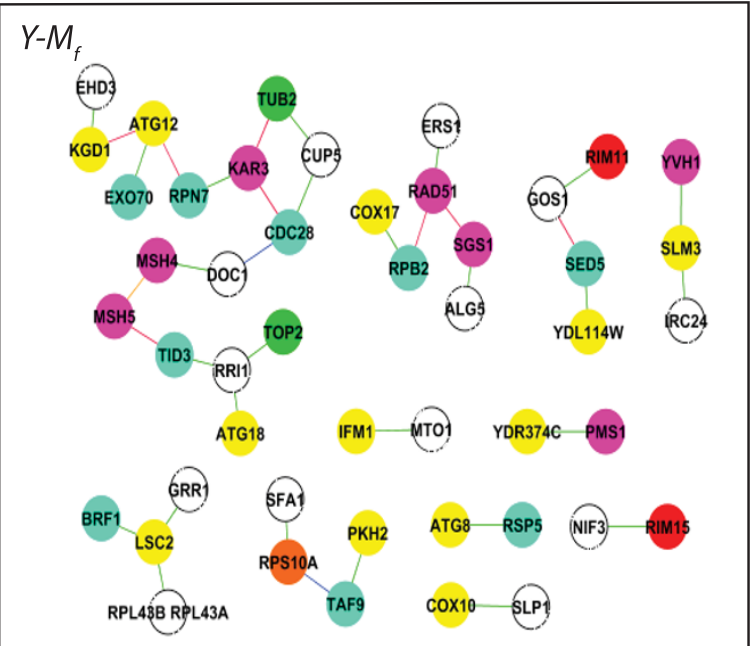

D

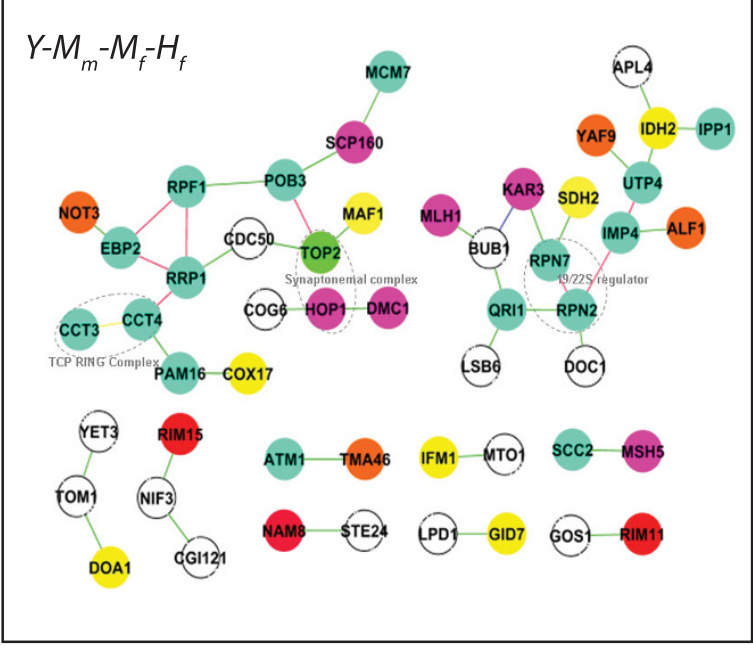

co-expression links

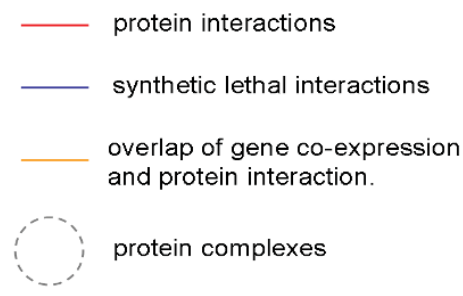

Figure 6 Conserved gene modules in meiotic prophase. Each conserved co-expression network was constructed by connecting the top-100 metagene pairs with the most significant P-values. Gene clusters containing either known yeast meiotic genes, sporulation-deficient genes, or sporulation-proficient genes were selected for display in the figure. Metagenes were labeled with yeast gene names. Metagenes and metagene connections were marked with different colors to represent information derived from yeast genomic datasets. A. $Y-M_{m}$ network. B. $Y-M_{f}$ network. C. $Y-H_{f}$ network. D. $Y-M_{m}-M_{f}-H_{f}$ network. 
Table 4 Genes annotated by meiosis term (GO:0007126) from the top-100 metagene pairs* in conserved co-expression networks

\begin{tabular}{|c|c|c|c|c|}
\hline & $Y-M_{m}$ & $Y-M_{f}$ & $Y-H_{f}$ & $Y-M_{m}-M_{f} H_{f}$ \\
\hline Yeast & $\begin{array}{l}\text { HOP1, KAR3, KAR4, MLH3, MSH4, MSH5, } \\
\text { NAM8, PCH2, RFA2, RIM15, SMC3, TOR1, } \\
\text { TUB2 }\end{array}$ & $\begin{array}{l}\text { KAR3, MSH4, MSH5, PMS1, RAD51, } \\
\text { RIM11, RIM15, SGS1, TOP2, TUB2, } \\
\text { YVH1 }\end{array}$ & $\begin{array}{l}\text { DMC1, HOP1, MSH5, RAD50, } \\
\text { RIM15, SCP160, SPO11, SPO22, } \\
\text { YHP1 }\end{array}$ & $\begin{array}{l}\text { DMC1, HOP1, KAR3, MLH1, } \\
\text { MSH5, NAM8, RIM11, RIM15, } \\
\text { SCP160, TOP2 }\end{array}$ \\
\hline Mouse & Mlh3, Msh4, Msh5, Smc3, Trip13 & Msh4, Msh5, Pms2, Rad51, Tex15 & - & Dmcl, Mlh1, Msh5 \\
\hline Human & - & - & DMC1, MSH5, RAD50, SPO11 & DMC1, MSH5 \\
\hline
\end{tabular}

RIM15, from gene co-expression networks that are known to control meiotic entry in yeast. Both showed sporulation deficiency when knocked out [25], suggesting that the mammalian orthologs of RIM11 and RIM15 might play a similar role in governing meiotic entry. Rim11 is a protein kinase required for signal transduction during meiotic entry in yeast. It promotes the formation of the Ime1-Ume6 complex by phosphorylating Ime 1 and Ume6 [45]. Glycogen synthase kinase-3b $(G s k-3 b)$ is the mammalian ortholog of yeast RIM11. Gsk-3b participates in a variety of cell signaling events in addition to regulating glycogen synthesis [46]. It is expressed in both spermatocytes and Sertoli cells in mice and rats. Further, inhibition of GSK-3b has been shown to prevent DNA replication in cultured rat germ cells [47], supporting our prediction that $G s k-3 b$ plays a role similar to that of its yeast ortholog, RIM11, in regulating meiotic entry in mammals. A co-expression link was observed between RIM11 and GOS1 in $Y-M_{f}$ and $Y$ $M_{m}-M_{f^{-}} H_{f}$. Yeast GOS1 and its mammalian ortholog, Gosr1, encode a SNARE protein that is involved in Golgi transport [48]. The connection between Golgi transport and meiotic entry is intriguing and previously has not been documented. RIM15 is a glucose-repressible protein kinase that has been identified as a regulator of $I M E 2$, a key gene that controls meiotic entry in yeast [49]. Mastl (microtubule-associated serine/threonineprotein kinase-like) is the mammalian ortholog of RIM15. RIM15 has a co-expression linkage to NIF3 in all four networks, suggesting that NIF3 may also be involved in regulation of meiotic entry. The function of yeast NIF3 is unknown [50]. Its mammalian ortholog Nif3l showed a ubiquitous expression pattern with encoded protein localized in the cytoplasm [51].

\section{Experimental validation of novel meiotic genes in prophase}

Our computational research generated many candidate meiotic genes that function during meiotic prophase (Figure 6). Here we focus on validating candidates that show co-expression links with recombination genes, chromatin cohesion genes, and genes involved in meiotic entry in at least two networks. By taking this criterion, we experimentally tested five genes, DOC1, STE24,
COG6, GOS1, and NIF3, plus YDR374C, an uncharacterized ORF co-expressed with PMS1 in only one network.

We conducted sporulation assays using yeast deletion strains of these genes (Table 5, Additional file 1, Figure S5). Homozygous diploid deletion strains have been systematically constructed for every yeast gene on the BY4743 genetic background [24]. At the end of the sporulation experiment, we calculated the percentages of cells completing meiosis I (binucleates+trinucleates +tetranucleates) and meiosis II (trinucleates+tetranucleates). Around 300 cells were analyzed for each strain $[4,5]$. Our positive control is the wild-type strain. Around $18 \%$ of the wild-type cells in the population went through meiosis I and II and then sporulated. This sporulation efficiency is consistent with previous observation on the BY4743 wild-type strain [24]. Our negative controls are deletion strains of rim11 and rim15; both are required for signal transduction during meiotic entry in yeast [25]. We observed that the sporulation process was completely blocked for these two deletion strains. Next, we tested the sporulation efficiency of the six candidate meiotic genes. We found that all of them showed sporulation defects, equivalent to a $100 \%$ validation rate. Three that are predicted to be involved in meiotic recombination (DOC1, STE24, and YDR374C) exhibited three- to six-fold reductions in sporulation efficiency. In particular, no tetranucleate cell was observed in the doc1 mutant. COG6, with a co-expression link to HOP1, showed a 1.4-fold decrease in sporulation efficiency. GOS1 and NIF3 exhibited co-expression linkages with RIM11 and RIM15, respectively. Deletion mutants of gos 1 and nif3 show 1.5- to 2.5- fold decreases in sporulation efficiency. The above experimental verification demonstrates that our conserved co-expression network provides a powerful tool for discovering novel meiotic genes. Mammalian orthologs of these genes can be further evaluated using a mouse system.

\section{Discussion}

Meiotic prophase is a critical stage in determining reproductive success, as errors in meiotic initiation and recombination can lead to chromosome mis-segregation. In fact, defects in meiotic chromosome segregation are the leading cause of miscarriages and one of the leading 
Table 5 Validation of predicted meiotic genes by sporulation assay

\begin{tabular}{cllll}
\hline & Strain & Gene & \% cells completing meiosis I* & \% cells completing meiosis II \\
\hline Positive control & BY4743 & - & $18.3 \%$ & $17.7 \%$ \\
\hline Negative control & BY4743 rim11 & RIM11 & $0 \%$ & $0 \%$ \\
& BY4743 rim15 & RIM15 & $0 \%$ & $0 \%$ \\
\hline Predicted meiotic genes & BY4743 doc1 & DOC1 & $3.2 \%$ & $0.4 \%$ \\
& BY4743 ste24 & STE24 & $5.3 \%$ & $4.7 \%$ \\
& BY4743 ydr374C & YDR374C & $4.5 \%$ & $4.5 \%$ \\
& BY4743 cog6 & COG6 & $13.6 \%$ & $13.0 \%$ \\
& BY4743 gos1 & GOS1 & $8.2 \%$ & $7.2 \%$ \\
& BY4743 nif3 & NIF3 & $12.4 \%$ & $10.9 \%$ \\
\hline
\end{tabular}

* Percentage of binucleates, trinucleates, and tetranucleates among 300 cells for each strain.

\# Percentage of trinucleates and tetranucleates among 300 cells for each strain.

causes of birth defects in humans [1]. Identification of germ-cell-specific meiotic genes in multicellular organisms is a complex task because gonads contain distinct types of cells, of which only a fraction are germ cells. Because human gonads are an intractable experimental system, human meiosis has mainly been investigated using traditional in vivo mouse genetics, which is timeconsuming and difficult to scale up. Only a limited number of mammalian meiotic genes have been characterized; for this reason, it is important to be able to predict novel meiotic genes in mammals.

In this study, we assembled cross-species and crosssex whole-genome expression profiles on meiotic prophase in yeast, mouse embryonic ovary, mouse postnatal testis, and human fetal ovary. We found significant enrichment of known meiotic genes in co-expression networks, suggesting the feasibility of our approach for inferring conserved meiotic genes in multiple species and between sexes. Indeed, conservation of co-expression between species improved the identification of mammalian meiosis genes. We further characterized coexpression pairs and demonstrated that they are functionally related. Most top-ranked co-expression genes are highly expressed, particularly in mammals. From coexpression networks, we identified genes important to the meiotic process in both yeast and mammals. Our results show that major recombination and cohesion proteins are conserved across species. We also identified mammalian orthologs of yeast meiotic genes RIM11 and RIM15 as candidates that might regulate meiotic entry in mammals. Co-expression links enabled us to infer roles for genes not previously found to function during the meiotic process. We experimentally validated six predicted meiotic genes using a genetically tractable yeast system, all of which exhibited sporulation defects. The mammalian orthologs of these new meiotic genes can be further tested using a mouse system. In contrast to the simple clustering of meiosis expression profiles which could pinpoint many meiotic gene candidates
$[6-9,15,16]$, our method quantifies conserved co-expression with P-values in four networks, which allows us to prioritize candidate genes and interactions for experimental testing.

We focused on validating candidate genes showing coexpression links with known meiotic genes. This criterion is likely to yield a high validation rate. Our future direction will include testing conserved co-expression metagene pairs common to multiple networks. This would very likely increase the list of candidate genes that function during conserved meiotic process. In our study, we used microarray profiles of testes and ovaries that closely represent in vivo germ cell gene expression. A complementary approach is to use expression data from isolated germ cells; such data is only available in males $[8,15]$. Although sorted germ cell samples removed most somatic cells, their expression patterns may have changed dramatically from in vivo status. The microarray studies use in our analysis were not particularly designed to capture transcriptional changes in meiotic prophase except for the data on human fetal ovary [6]. The yeast experiments covered the entire sporulation process [5], while the mouse experiments captured the developmental process of the murine embryonic gonad and the first wave of spermatogenesis in postnatal testis $[8,9]$. Therefore, only limited time points were included to describe meiotic prophase. Our approach will likely become more valuable for the identification of novel genes when expression data are available to capture detailed transcriptional changes during meiotic prophase. This will enable us to better understand the genetic controls that regulate meiotic entry and progression in this critical developmental stage.

\section{Conclusions}

We constructed conserved co-expression networks for meiotic prophase by integrating cross-species and crosssex expression profiles from budding yeast, mouse, and human. The co-expression links in the networks 
confirmed known meiotic genes and identified several novel genes that might be critical players in meiosis in multiple species. Indeed, the conserved co-expression approach improved the identification of mammalian meiotic genes. Six candidate genes were subsequently validated in the yeast and all showed sporulation defects. These results suggest our approach is highly efficient to identify evolutionarily conserved gene modules and novel genes in meiotic prophase.

\section{Methods}

\section{Metagene construction}

Pairwise ortholog groups of yeast, mouse, and human were downloaded from Inparanoid, a database of eukaryotic orthologs [52]. Only seed orthologs found through a reciprocal best match between two genomes were kept for metagene construction. Three types of metagenes were obtained by identifying orthologs conserved either across all three species (YMH) or only between two species (YM, YH) (Table 1). Three metagene types are mutually exclusive. Most metagenes contain a single gene from each organism. Each gene was assigned to only one metagene.

\section{Microarray expression profiles}

Four time-series microarray studies were selected to investigate global gene expression of meiotic prophase in yeast, mouse postnatal testis (GSE12769), mouse embryonic ovary (GSE6916), and human fetal ovary (GSE15431) [5,6,8,9]. These experiments all used the Affymetrix microarray platform. For the yeast experiment, gene expression was monitored using aliquots of SK1 cells at $0,1,2,3,4,6,8$ and 10 hours after transfer of cells to sporulation medium [5]. For microarray profiling of the first wave of spermatogenesis, duplicate testis samples were obtained from postnatal mice at ages of $0,3,6,8,10,14,18,20,30,35$, and 56 days postpartum [8]. For female mice, duplicate samples of embryonic ovaries were collected at 11.5, 12.5, 14.5, 16.5, and 18.5 days of postcoitum [9]. For female humans, ovaries from fetuses at 9.1, 9.6, 11, 12, 12.9, 13.6, 13.9, 14.4, $16.1,16.4,16.9,17.1$, and 18.1 weeks of gestation were obtained; each time-point was represented by one fetus sample except there were three samples at 9.6 weeks, two at 13.6 weeks and two at 16.9 weeks [6]. Although the human female microarray captures the timeframe of meiotic prophase, the yeast and male mouse microarrays cover the entire meiosis, and the female mouse experiment was designed to capture the development of the murine embryonic ovary. To consistently identify changes in gene expression during meiotic prophase, only time points within meiotic prophase were considered in our analysis (0-4 hours for yeast, 6-14 days postpartum for mouse postnatal testis, 11-14 days postcoitum for mouse embryonic ovary, 9-18 weeks gestation for human fetal ovary).

Microarray data were normalized to the mean or median of each array, as described in the original papers $[5,6,8,9]$. For human and mouse experiments, unique probes that map to the same gene were averaged to obtain the gene signal intensity. If unique probes do not exist for a gene, we averaged signal intensity of probes with a "_a" suffix. Duplicate samples at each time point were averaged. The maximal signal intensity over the course of prophase was identified for each gene in each microarray. We used this signal intensity to define gene expression levels. The top $90 \%$ of highly expressed genes in each array were used to calculate Pearson correlations of gene pairs, while the bottom $10 \%$ of lowly expressed genes were removed from further study. This prevented the introduction of very lowly expressed genes into the conserved co-expression network. We did this because Pearson correlations measure similarity of gene expression rather than absolute levels, and lowly expressed gene profiles are often dominated by noise.

\section{Conserved gene co-expression networks}

Pearson correlations, $\mathrm{rxy}=\sum \mathrm{i}=1 \mathrm{n}\left(\mathrm{xi}-\mathrm{x}^{-}\right)\left(\mathrm{yi}-\mathrm{y}^{-}\right)(\mathrm{n}-1) \mathrm{SxSy}$, were calculated for gene pairs belonging to metagenes across the prophase time points in each microarray. Here $x$ and $y$ are expression data vectors of length $n$ for two genes, $\mathrm{x}^{-}$and $\mathrm{y}^{-}$are means, and $s_{x}$ and $s_{y}$ are standard deviations. If a metagene contains more than one gene in a species, Pearson correlations for metagene pairs were computed by averaging multiple gene-gene Pearson correlations. Otherwise, Pearson correlations for metagene pairs are the same as correlations for gene pairs. Metagene pairs in the same species were ranked by their Pearson correlations. Then each metagene pair was associated with a rank ratio, $r$, which was the rank divided by the total number of metagene pairs in a species $s$. We computed the P-value for each metagene pair across a total of $n$ species from the joint cumulative distribution of an $n$-dimensional order statistics [19]: $\mathrm{P}$ $(\mathrm{r} 1 \mathrm{r} 2, \ldots, \mathrm{rn})=\mathrm{n} ! \int 0 \mathrm{r} 1 \int \mathrm{s} 1 \mathrm{r} 2 \ldots . \mathrm{sn}-1 \mathrm{rnds} 1 \mathrm{ds} 2 \ldots \mathrm{dsn}$, where $r_{1}$ $<r_{2} \ldots<\mathrm{r}_{n}$. This $\mathrm{P}$-value quantifies the evolutionarily conserved co-expression of metagene pairs. Metagene pairs with P-values greater than a threshold can be connected to form conserved co-expression networks.

\section{Hierarchical clustering for identifying conserved co- expression}

An alternative to our approach is to cluster Pearson correlation matrix to identify conserved co-expression. Pearson correlation of metagene pairs was calculated for each species. The pairwise correlation matrices for different species can be merged into a consensus correlation matrix by taking the minimum correlation for each 
metagene pair. The resulting consensus matrix was converted into a dissimilarity matrix and subjected to average linkage hierarchical clustering algorithms. The dynamic tree cut method was applied to define branches as co-expression modules (minClusterSize $=40)$ [53]. Order statistics and hierarchical clustering perform similarly in identifying known meiotic genes (Additional file 1, Table S5). The advantage of order statistics over hierarchical clustering is that it defines pairwise gene coexpression thus can prioritize genes for experimental testing.

\section{GO term enrichment}

Significantly shared GO terms are used to describe common functions of a query gene set. To determine whether any GO terms are enriched in a query gene list at a frequency greater than that would be expected by chance, we calculated the probability from a hypergeometric distribution: $\mathrm{p}(\mathrm{x} \geq \mathrm{k})=\sum \mathrm{x}=\mathrm{kmin}(\mathrm{m}, \mathrm{n}) \mathrm{C}(\mathrm{m}, \mathrm{x}) \mathrm{C}(\mathrm{t}-\mathrm{m}$, $\mathrm{n}-\mathrm{x}) / C(\mathrm{t}, \mathrm{n})$ where $C(j, k)$ is the combinatorial factor $j ! / k !$ $(j-k) !$. In this equation, $t$ is the total number of genes in a network, $n$ is the number of genes in the network that are annotated by a GO term, $m$ is the number of genes in a query list (for example, genes in top-100 metagene pairs), and $k$ is the number of genes in that list which are annotated by the GO term.

\section{GO semantic similarity}

The method to calculate GO semantic similarity was described in the reference [23]. Specifically, each node in the GO tree is associated with a probability, $p(c)$, representing the chance a concept occurs on the node or any of its children. Thus the probability increases as we move up toward the root of GO tree, where the probability is 1 . To calculate the semantic similarity for a pair of genes, the minimal $p(c)$ of parental nodes shared by two genes will be identified. The similarity score is defined as the negative $\log 10$ of the minimal $p$ (c). Because each gene pair is associated with a semantic similarity score, the averaged GO semantic similarity is the mean of similarity scores of a group of gene pairs. The averaged GO semantic similarity can be used to quantify the overall functional association of a group of gene pairs.

\section{Yeast sporulation assays}

Wild-type yeast strain BY4743 and homozygous diploid deletion strains on the BY4743 background (isogenic to the strain S288c) were patched onto a GNA pre-sporulation plate. The GNA plate was incubated at $30^{\circ} \mathrm{C}$ overnight. Cells were transferred to the sporulation medium and incubated on a shaker at $25^{\circ} \mathrm{C}$ for 5 days [24]. Approximately $0.1 \mathrm{OD}$ cells were fixed with $70 \%$ ethanol for 30 minutes. Samples were washed with PBS twice, and then stained with 2uM Hoechst 33342 (H1399 Invitrogen) at room temperature for 30 minutes. Cells containing tetranucleate, trinucleate, binucleate, and mononucleate were counted from a total of 300 cells for each strain using a Zeiss 510 Meta multiphoton confocal microscope.

\section{Additional material}

Additional file 1: Supplementary figures and tables. This file includes five supplementary figures and five supplementary tables.

\section{Acknowledgements}

The authors would like to acknowledge Terry Hassold, Patricia Hunt, Michael Griswold, and Christopher Small for helpful discussions, and Lizhong Yang for providing time-series expression profiles on mouse postnatal testis, mouse embryonic ovary, and human fetal ovary. The authors also wish to thank Xuewen Pan for providing homozygous diploid yeast deletion strains. This work was supported by a Washington State University start-up grant to PY.

\section{Author details}

${ }^{1}$ School of Molecular Biosciences, Washington State University, PO Box 647520, Pullman, WA 99164, USA. ${ }^{2}$ Center for Reproductive Biology, Washington State University, Pullman, WA 99164, USA. ${ }^{3}$ Department of Statistics, Washington State University, Pullman, WA 99164, USA.

\section{Authors' contributions}

$\mathrm{YL}$ and PY carried out the computational studies. $\mathrm{KL}$ carried out the sporulation assays. ND helped with the order statistics. PY conceived of and supervised the project. PY wrote the paper. All authors read and approved the final manuscript.

Received: 16 February 2010 Accepted: 6 September 2010 Published: 6 September 2010

\section{References}

1. Marston AL, Amon A: Meiosis: cell-cycle controls shuffle and deal. Nat Rev Mol Cell Biol 2004, 5(12):983-997.

2. Roeder GS, Bailis JM: The pachytene checkpoint. Trends Genet 2000, 16(9):395-403.

3. Chu S, DeRisi J, Eisen M, Mulholland J, Botstein D, Brown PO, Herskowitz I: The transcriptional program of sporulation in budding yeast. Science 1998, 282(5389):699-705.

4. Friedlander G, Joseph-Strauss D, Carmi M, Zenvirth D, Simchen G, Barkai N: Modulation of the transcription regulatory program in yeast cells committed to sporulation. Genome Biol 2006, 7(3):R20.

5. Primig M, Williams RM, Winzeler EA, Tevzadze GG, Conway AR, Hwang SY, Davis RW, Esposito RE: The core meiotic transcriptome in budding yeasts. Nat Genet 2000, 26(4):415-423.

6. Houmard B, Small C, Yang L, Naluai-Cecchini T, Cheng E, Hassold T, Griswold M: Global gene expression in the human fetal testis and ovary. Biol Reprod 2009, 81(2):438-443.

7. Schultz N, Hamra FK, Garbers DL: A multitude of genes expressed solely in meiotic or postmeiotic spermatogenic cells offers a myriad of contraceptive targets. Proc Natl Acad Sci USA 2003, 100(21):12201-12206.

8. Shima JE, McLean DJ, McCarrey JR, Griswold MD: The murine testicular transcriptome: characterizing gene expression in the testis during the progression of spermatogenesis. Biol Reprod 2004, 71(1):319-330.

9. Small CL, Shima JE, Uzumcu M, Skinner MK, Griswold MD: Profiling gene expression during the differentiation and development of the murine embryonic gonad. Biol Reprod 2005, 72(2):492-501.

10. Bowles J, Koopman P: Retinoic acid, meiosis and germ cell fate in mammals. Development 2007, 134(19):3401-3411. 
11. Hunt PA, Hassold TJ: Human female meiosis: what makes a good egg go bad? Trends Genet 2008, 24(2):86-93.

12. Hassold T, Hall H, Hunt P: The origin of human aneuploidy: where we have been, where we are going. Hum Mol Genet 2007, 16(Spec No. 2): R203-208.

13. Vallente RU, Cheng EY, Hassold TJ: The synaptonemal complex and meiotic recombination in humans: new approaches to old questions. Chromosoma 2006, 115(3):241-249.

14. Schlecht U, Primig M: Mining meiosis and gametogenesis with DNA microarrays. Reproduction 2003, 125(4):447-456.

15. Chalmel F, Rolland AD, Niederhauser-Wiederkehr C, Chung SS, Demougin $P$, Gattiker A, Moore J, Patard JJ, Wolgemuth DJ, Jegou B, et al: The conserved transcriptome in human and rodent male gametogenesis. Proc Natl Acad Sci USA 2007, 104(20):8346-8351.

16. Olesen C, Nyeng P, Kalisz M, Jensen TH, Moller M, Tommerup N, Byskov AG: Global gene expression analysis in fetal mouse ovaries with and without meiosis and comparison of selected genes with meiosis in the testis. Cell Tissue Res 2007, 328(1):207-221.

17. Sharan R, Suthram S, Kelley RM, Kuhn T, McCuine S, Uetz P, Sittler T, Karp RM, Ideker T: Conserved patterns of protein interaction in multiple species. Proc Natl Acad Sci USA 2005, 102(6):1974-1979.

18. Slonim N, Elemento O, Tavazoie S: Ab initio genotype-phenotype association reveals intrinsic modularity in genetic networks. Mol Syst Biol 2006, 2, 20060005.

19. Stuart JM, Segal E, Koller D, Kim SK: A gene-coexpression network for global discovery of conserved genetic modules. Science 2003, 302(5643):249-255.

20. Ashburner M, Ball CA, Blake JA, Botstein D, Butler H, Cherry JM, Davis AP, Dolinski K, Dwight SS, Eppig JT, et al: Gene ontology: tool for the unification of biology. The Gene Ontology Consortium. Nat Genet 2000, 25(1):25-29.

21. Mewes HW, Dietmann S, Frishman D, Gregory R, Mannhaupt G, Mayer KF, Munsterkotter M, Ruepp A, Spannagl M, Stumpflen V, et al: MIPS: analysis and annotation of genome information in 2007. Nucleic Acids Res 2008, 36 Database: D196-201.

22. Stark C, Breitkreutz BJ, Reguly T, Boucher L, Breitkreutz A, Tyers M: BioGRID: a general repository for interaction datasets. Nucleic Acids Res 2006, , 34 Database: D535-539.

23. Lord PW, Stevens RD, Brass A, Goble CA: Investigating semantic similarity measures across the Gene Ontology: the relationship between sequence and annotation. Bioinformatics 2003, 19(10):1275-1283.

24. Giaever G, Chu AM, Ni L, Connelly C, Riles L, Veronneau S, Dow S, LucauDanila A, Anderson K, Andre B, et al: Functional profiling of the Saccharomyces cerevisiae genome. Nature 2002, 418(6896):387-391.

25. Deutschbauer AM, Williams RM, Chu AM, Davis RW: Parallel phenotypic analysis of sporulation and postgermination growth in Saccharomyces cerevisiae. Proc Natl Acad Sci USA 2002, 99(24):15530-15535.

26. Jin YH, Dunlap PE, McBride SJ, Al-Refai H, Bushel PR, Freedman JH: Global transcriptome and deletome profiles of yeast exposed to transition metals. PLoS Genet 2008, 4(4):e1000053.

27. Ye P, Peyser BD, Pan X, Boeke JD, Spencer FA, Bader JS: Gene function prediction from congruent synthetic lethal interactions in yeast. Mol Syst Biol 2005, 1, 20050026.

28. Tong $A H$, Lesage $G$, Bader GD, Ding $H, X u H$, Xin $X$, Young J, Berriz GF, Brost $R L$, Chang $M$, et al: Global mapping of the yeast genetic interaction network. Science 2004, 303(5659):808-813.

29. Bader GD, Betel D, Hogue CW: BIND: the Biomolecular Interaction Network Database. Nucleic Acids Res 2003, 31(1):248-250.

30. Salwinski L, Miller CS, Smith AJ, Pettit FK, Bowie JU, Eisenberg D: The Database of Interacting Proteins: 2004 update. Nucleic Acids Res 2004, , 32 Database: D449-451

31. Chatr-aryamontri A, Ceol A, Palazzi LM, Nardelli G, Schneider MV, Castagnoli L, Cesareni G: MINT: the Molecular INTeraction database. Nucleic Acids Res 2007, , 35 Database: D572-574.

32. Kerrien S, Alam-Faruque $Y$, Aranda B, Bancarz I, Bridge A, Derow C, Dimmer E, Feuermann M, Friedrichsen A, Huntley R, et al: IntAct-open source resource for molecular interaction data. Nucleic Acids Res 2007, 35 Database: D561-565.

33. Keshava Prasad TS, Goel R, Kandasamy K, Keerthikumar S, Kumar S, Mathivanan S, Telikicherla D, Raju R, Shafreen B, Venugopal A, et al: Human
Protein Reference Database-2009 update. Nucleic Acids Res 2009, , 37 Database: D767-772

34. Schlabach MR, Luo J, Solimini NL, Hu G, Xu Q, Li MZ, Zhao Z Smogorzewska A, Sowa ME, Ang $X \mathrm{~L}$, et al: Cancer proliferation gene discovery through functional genomics. Science 2008, 319(5863):620-624

35. Silva JM, Marran K, Parker JS, Silva J, Golding M, Schlabach MR, Elledge SJ, Hannon GJ, Chang K: Profiling essential genes in human mammary cells by multiplex RNAi screening. Science 2008, 319(5863):617-620.

36. Liao BY, Zhang J: Null mutations in human and mouse orthologs frequently result in different phenotypes. Proc Natl Acad Sci USA 2008, 105(19):6987-6992

37. Ciosk R, Shirayama M, Shevchenko A, Tanaka T, Toth A, Nasmyth K: Cohesin's binding to chromosomes depends on a separate complex consisting of Scc2 and Scc4 proteins. Mol Cell 2000, 5(2):243-254.

38. Passmore LA, McCormack EA, Au SW, Paul A, Willison KR, Harper JW, Barford D: Doc1 mediates the activity of the anaphase-promoting complex by contributing to substrate recognition. EMBO J 2003 , 22(4):786-796

39. Grossberger R, Gieffers C, Zachariae W, Podtelejnikov AV, Schleiffer A, Nasmyth K, Mann M, Peters JM: Characterization of the DOC1/APC10 subunit of the yeast and the human anaphase-promoting complex. $J$ Biol Chem 1999, 274(20):14500-14507.

40. Nakagawa T, Ogawa $\mathrm{H}$ : Involvement of the MRE2 gene of yeast in formation of meiosis-specific double-strand breaks and crossover recombination through RNA splicing. Genes Cells 1997, 2(1):65-79.

41. Tam A, Nouvet FJ, Fujimura-Kamada K, Slunt H, Sisodia SS, Michaelis S: Dual roles for Ste $24 p$ in yeast a-factor maturation: $\mathrm{NH} 2$-terminal proteolysis and COOH-terminal CAAX processing. J Cell Biol 1998, 142(3):635-649.

42. Freije JM, Blay P, Pendas AM, Cadinanos J, Crespo P, Lopez-Otin C: Identification and chromosomal location of two human genes encoding enzymes potentially involved in proteolytic maturation of farnesylated proteins. Genomics 1999, 58(3):270-280.

43. Hardwick KG, Pelham HR: SED5 encodes a 39-kD integral membrane protein required for vesicular transport between the ER and the Golgi complex. J Cell Biol 1992, 119(3):513-521.

44. Williamson MS, Game JC, Fogel S: Meiotic gene conversion mutants in Saccharomyces cerevisiae. I. Isolation and characterization of pms1-1 and pms1-2. Genetics 1985, 110(4):609-646.

45. Malathi K, Xiao Y, Mitchell AP: Interaction of yeast repressor-activator protein Ume6p with glycogen synthase kinase 3 homolog Rim11p. Mol Cell Biol 1997, 17(12):7230-7236.

46. Cohen P, Frame S: The renaissance of GSK3. Nat Rev Mol Cell Biol 2001, 2(10):769-776

47. Guo TB, Chan KC, Hakovirta H, Xiao Y, Toppari J, Mitchell AP, Salameh WA: Evidence for a role of glycogen synthase kinase-3 beta in rodent spermatogenesis. J Androl 2003, 24(3):332-342.

48. McNew JA, Coe JG, Sogaard M, Zemelman BV, Wimmer C, Hong W, Sollner TH: Gos1p, a Saccharomyces cerevisiae SNARE protein involved in Golgi transport. FEBS Lett 1998, 435(1):89-95.

49. Vidan S, Mitchell AP: Stimulation of yeast meiotic gene expression by the glucose-repressible protein kinase Rim15p. Mol Cell Biol 1997, 17(5):2688-2697.

50. Reinders J, Zahedi RP, Pfanner N, Meisinger C, Sickmann A: Toward the complete yeast mitochondrial proteome: multidimensional separation techniques for mitochondrial proteomics. J Proteome Res 2006, 5(7):1543-1554.

51. Tascou S, Uedelhoven J, Dixkens C, Nayernia K, Engel W, Burfeind P: Isolation and characterization of a novel human gene, NIF3L1, and its mouse ortholog, Nif3I1, highly conserved from bacteria to mammals. Cytogenet Cell Genet 2000, 90(3-4):330-336.

52. Berglund AC, Sjolund E, Ostlund G, Sonnhammer EL: InParanoid 6: eukaryotic ortholog clusters with inparalogs. Nucleic Acids Res 2008, , 36 Database: D263-266.

53. Langfelder $\mathrm{P}$, Horvath $\mathrm{S}$ : Eigengene networks for studying the relationships between co-expression modules. BMC Syst Biol 2007, 1:54.

doi:10.1186/1752-0509-4-125

Cite this article as: Li et al:: A yeast's eye view of mammalian reproduction: cross-species gene co-expression in meiotic prophase. BMC Systems Biology 2010 4:125 